\title{
Planning decision alterations and container terminal efficiency
}

\author{
Buddhi A. Weerasinghe and $\mathrm{H}$. Niles Perera \\ Center for Supply Chain, Operations and Logistics Optimization, \\ University of Moratuwa, Moratuwa, Sri Lanka and \\ Professor H.Y. Ranjit Perera Institute for Applied Research, \\ Nugegoda, Sri Lanka, and \\ Phillip Kießner \\ Chair of Enterprise Logistics, Technische Universität Dortmund, \\ Dortmund, Germany
}

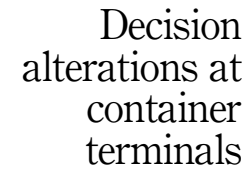

65

Received 24 April 2021 Revised 4 August 2021 12 November 2021 3 January 2022

Accepted 11 January 2022

\begin{abstract}
Purpose - This paper examines how the altering nature of planning decisions affects operational efficiency in seaport container terminals. The uncertainty and the role of the planner were investigated considering the dynamic integrated planning function of the quay to yard interface.

Design/methodology/approach - A system dynamics model has been built to illustrate the integrated dynamic environment. Data collection was conducted at a leading container terminal at a hub port. The model was simulated for different scenarios to derive findings.

Findings - The planner has been identified as the agent who makes alterations between the initial operational plan and the actual plan. The initial plan remains uncertain even when there is no impact from crane breakdowns, requiring a significant number of alterations to be made. The planner who had worked on the yard plan had altered (approximately 45\%) the initial plan than the alterations done by the planner who had worked on the vessel plan. As a result, the feedback loop that is created by the remaining moves at each hourly operation influences the upcoming operation as much as crane breakdowns influence.

Originality/value - The uncertainty and the role of the planner were investigated considering the dynamic integrated planning function of the quay to yard interface. The findings of this study are significant since terminal efficiency is examined considering the quayside and landside as an integrated system.
\end{abstract}

Keywords Container terminal planning, System dynamics, Planning decisions, Crane breakdowns, Terminal planner, Uncertainty

Paper type Research paper

\section{Introduction}

Maritime trade is a vast industry and seaports are the most vital component within that. Approximately $70 \%$ of the cargo is handled by global seaborne container trade in terms of value (Dadashi et al., 2017). According to the Review of Maritime Transport 2020, UNCTAD (United Nations Conference on Trade and Development), the annual growth of maritime trade has fallen by $4.1 \%$ in 2020 due to the COVID-19 pandemic (UNCTAD, 2020). However, the annual average growth of the seaborne trade for the period between 2019-2024 has been predicted to be $3.4 \%$ (UNCTAD, 2019). Global container traffic growth for 2020 has been predicted as $4.7 \%$ before the crisis arose (UNCTAD, 2019). The annual average growth of the containerized trade between 2019 and 2024 has been predicted as 4.5\% (UNCTAD, 2019). Seaport terminals are specified for different tasks based on the cargo they handle. The focus of this study is the planning side of handling containerized cargo in seaport terminals.

\subsection{Planning function in container terminal operations}

Container terminal operations planning is extremely complex since global shipping lines are demanding their needs at a competitive price in a dynamic environment. Most of the

(C) Pacific Star Group Education Foundation. Licensed re-use rights only.

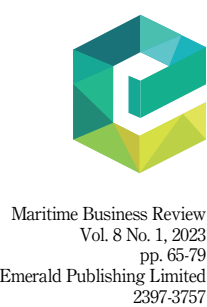

DOI 10.1108/MABR-04-2021-0035 
MABR

8,1

\section{6}

international ports invest in port community systems, port call optimization and automation projects to provide a better service (Pham et al., 2020). Consequently, it is important to understand the role of the planning function in a dynamic environment. One of the major focuses of this study is to investigate the integrated planning function of seaport container terminals to avoid the traditional approach of investigating individual planning functions in isolation. The impact of planning alterations on container terminal productivity at the operational level will be explored in this paper.

\subsection{Structure of the paper}

The literature review presents an overview of the current situation in theory and practice (section 2). Hypotheses that were derived based on the identified research gaps are discussed in subsection 2.7. Section 3 explains reasons for selecting system dynamics (SD) as the method and its procedure. Section 4 portrays the analysis that was conducted using extracted data from a terminal operating system (TOS) as well as relevant observations and discussions. A systematic approach is used to understand planning alterations and the eventual impact on terminal efficiency as presented in section 4 . Section 5 elaborates the results of the analysis and findings. Section 6 summarizes the study by introducing future research directions.

\section{Literature review}

\subsection{Quayside planning}

The vessel operation is handled at the quayside. Berth allocation problem (BAP), quay crane assignment (QCA), quay crane (QC) scheduling and the vessel stowage planning are the specific planning tasks at the quayside (Nishimura, 2019). The focus on the quayside was less than the focus on the landside when evaluating previous studies in the last decade (2011-2020) (Kizilay and Eliiyi, 2020). The time taken for the vessel operation is calculated using the number of QC moves to be completed. Crane intensity is calculated by identifying the accurate and optimized number of cranes that can be deployed (optimal QC option) to the vessel. The stowage plan contains information about containers that are planned to be moved. Information such as weight, destination port and further information of the containers can be identified in the initial plan (Beens and Ursavas, 2016). In the QCA problem, the number of QCs that are deployed to the relevant vessel is decided. Those cranes are deployed to the relevant vessel bay positions (Chen et al., 2012). Minimizing the total movements of each crane is a crucial constraint (Dulebenets, 2017). Berth planner's focus can be identified within the focus of arranging the "berth window" that includes berth allocation and schedules. The vessel planner can be identified as the person who plans the loading operation.

\subsection{Landside planning}

Landside operations are carried out at the container yard. A yard is a plot where containers are temporarily stacked until those are loaded to their outbound vessels or delivered to the relevant consignees or stakeholders (Gunawardhana et al., 2021). Yard management is a tactical-level decision process in container terminals (Zhen et al., 2016). Yard space allocation (YSA), yard crane (YC) deployment, YC scheduling and reshuffling operations are the major planning tasks within yard management (Jin et al., 2014). A recent study reported that dynamic approaches are more convenient than conventional approaches (Görges and Freitag, 2020). Gunawardhana et al. (2021) prove dynamic approaches are more convenient while Kizilay and Eliiyi (2020) have mentioned the same. YSA deals with the problem of allocating suitable space for containers following the stacking policies of the terminal. Stacking policies can be adjusted to match its capacity levels (Stahlbock and Voß, 2007). The number of container moves, travel distance of trucks, the completion time of tasks, yard space utilization, $\mathrm{YC}$ and $\mathrm{QC}$ utilization and 
operational costs are the key metrics in yard operations (Kizilay and Eliiyi, 2020). Usually, the identified YC option is 1.5 per QC operation (Mar-Ortiz et al., 2019). Due to this requirement, YC operation for loading should start in advance, and $\mathrm{YC}$ is dedicated to a yard subblock in the loading process (Zhen et al., 2016). YC system connects the quayside and landside operations (Nishimura, 2019). Most common YC equipment such as rubber tired gantry cranes and rail mounted gantry cranes are needed for the operations. Yard truck (YT) scheduling is always integrated with both quayside planning and landside operations that are planned by both, the vessel and the yard planners (He et al., 2019). YT scheduling is a part of real-time systems nowadays which is monitored by operations controllers under the supervision of planners.

\subsection{Integrated approach in terminal planning}

As Kizilay and Eliiyi (2020) illustrate, even integrated studies have not provided an overarching image to get an understanding of the dynamic behavior as Figure 1 illustrates. Moreover, previous studies have not focused on the integrated planning perspective sufficiently. Kizilay and Eliiyi (2020) evaluate more than 200 preceding studies (2011-2020) and find that the percentage of studies focusing on the integrated perspective is less than $15 \%$. Integration is the application side of TOS in seaport terminal planning. Legato and Mazza (2019) have mentioned that integrated determination of the right number of trucks between quayside and yard is a key consideration for planning. This integrated focus is more efficient than the freestanding is an obvious fact in container terminal planning.

\subsection{Planner role inside terminal planning}

Planners can be identified as decision-makers at tactical and operational levels. Making the plan with relevant decisions for the aforementioned planning functions is the key task that a terminal planner is entrusted with. Once the plan is complete for operation, the actual operation should be monitored and the relevant alterations should be applied (Böse, 2020). However, this dynamic planning role has not been investigated in previous studies sufficiently. Human resource management practices have been discussed from Legato and Monaco (2004) to Lindroth et al. (2020) as a separate research domain. The study, "Relating planner task performance for container terminal operations to multi-tasking skills and personality type", that has been published by Kurapati et al. (2017) is a watershed study focusing on the role of a terminal planner. Only less than five studies have been found related to this specific scope while perusing the literature. Importantly, any study related to the planner behavior was not found within the dynamic planning function in seaport container terminals.

\subsection{Uncertainty in terminal planning}

Although handling time can be calculated, there is no assurance that the allocated tasks will be accomplished within the calculated time (He et al., 2019). Vessel handling time is not a fixed

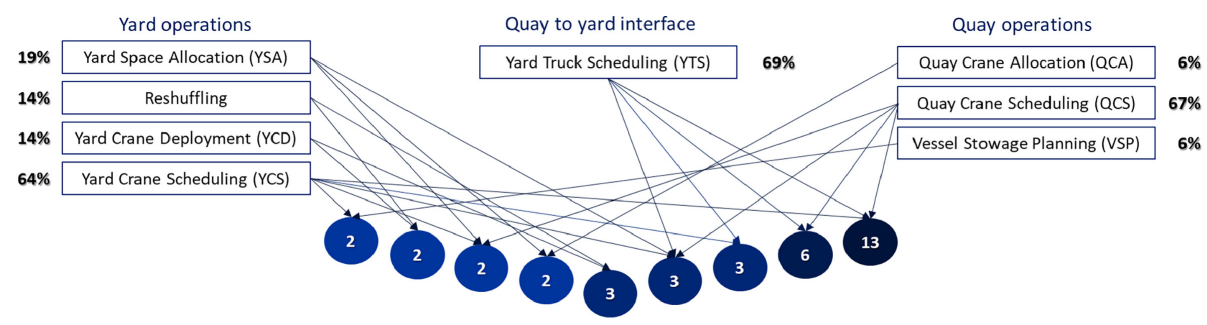

Source(s): Kizilay and Eliiyi (2020)

\section{Decision alterations at container terminals}


MABR

8,1

68

value, but a stochastic parameter (Zhou et al., 2016). Understanding those uncertainties in planning in the early stage is important (Zhou et al., 2016). The reason behind this is the integrated nature of terminal planning (subsection 2.3). Breakdowns or disruptions of cranes are one of the reasons behind uncertainties that alter the plan (He et al., 2019). This includes breakdowns of all types of cranes in seaports - regardless of whether QCs or YCs. All the breakdowns are recorded in TOS where the capability to revise the operation after the breakdowns is available (Navis LLC, 2014). Kavoosi et al. (2019) have stated that understanding the uncertainty in handling time requires future exploration. Böse (2020) has mentioned that bottlenecks that occur randomly (e.g. crane breakdowns) cannot be foreseen.

\subsection{Approach through planning alterations}

The first draft of the plan is no longer valid when the vessel is berthed, and the operations begin (Böse, 2020). Further breakdowns create a delay in the system and that should be mitigated using available options until the equipment becomes operational again. This is where the planner's role is more important. These dynamics have been discussed in previously (Gunawardhana et al., 2021). However, the focus is still on separate planning functions (Kizilay and Eliiyi, 2020). Prior works (Kurapati et al., 2017; Gunawardhana et al., 2021) have not approached dynamic problems through planning alterations similar to the gap that was found under integrated problems according to our extensive literature search. Therefore, our work brings a new approach through planning alterations into a trending area of integrated planning research that investigates dynamic planning.

\subsection{Research gaps and objectives}

As subsection 2.5 posits, disruptions in operation can be identified as the key factor in an uncertain environment. Predominantly, understanding the crane breakdown factor in studies that have been conducted on seaport container terminal planning is a research gap that is identified. Moreover, identifying the significance of crane breakdowns in altering planning decisions is one of the objectives of this study.

An insufficient understanding of the planner's role in the terminal planning task was identified as the second research gap under subsection 2.4. Along with, understanding the dynamic planning role while altering planning decisions is the second objective of this study.

Limited appreciation of the integrated planning perspective in extant studies on seaport container terminal planning was identified as the final research gap based on subsection 2.3. Understanding the impact of altering the initial plan on terminal efficiency is the last objective that was derived.

\section{Methodology}

\subsection{System dynamics (SD)}

The study considers alterations of planning decisions that display characteristics such as loop connectivity, feedback system thinking, cyclical behavior and loop integration. The whole idea of selecting a methodology is to understand the integrated planning function within the dynamic environment of terminal operations. Therefore, the methodology should be capable of understanding the dynamics of the system considering the integration of the system. Previous studies have used operations research techniques, systematic literature reviews and genetic algorithms to understand this dynamic (Kizilay and Eliiyi, 2020). However, those methodologies have not investigated the integrated aspect by considering all the planning functions within a selected time frame. Therefore, SD was selected as the methodology for this study. Macro perspective, ability to create feedback systems, having 
cyclical loops, loop integration, dynamic processes and integrated nature are major characteristics of SD that aligned with the research objectives. The work of Sterman (2000), Systems Thinking and Modeling for a Complex World, is a seminal resource for SD. This book was the key resource in developing the theory part of the methodology of this study.

\subsection{Applying SD and data collection}

Sterman (2000) has introduced five major steps to follow in SD: problem articulation, formulation of dynamic hypothesis, formulation of the simulation model, testing, policy design and evaluation. A seaport container terminal (2-4 $\mathrm{m}$ twenty-foot equivalent unit (TEU)s category) that is located at a major transshipment hub port in Asia was selected to ensure that the findings have some degree of generalizability. The terminal operates at more than twice its design capacity with three berths. Seventeen QCs and more than forty RTGs are being operated to cater to this capacity. The shift-based teams work for $12 \mathrm{~h}$ at a stretch. Thus, the working roster per day is completed by two teams that are working one after another allowing rest for the other team. All three groups were considered in the data collection. Data points for the actual operation were extracted from the TOS encompassing each hour. Planned data points were noted down before each hourly operation began. Data points were classified into each vessel (berth) operation and yard block operation based on their origin and destination when the data points were extracted. Although over 16,000 data points were collected from the planned operation, less than 16,000 data points were collected from the actual operation since the actual operation was behind schedule in the considered scenario.

\subsection{Applying SD and initial analysis}

In the first step, the identified problem has been expressed through several sub-steps. As the first sub-step, identifying what the problem is and identifying why the problem occurs is critical (Sterman, 2000). The literature review was relied on to answer these questions. However, this was further clarified through expert opinions collected at the selected container terminal and academics with an understanding of their operations. The problem was articulated considering three main areas: the theme of problem, key variables and time horizon (Jayalath and Perera, 2021). Planning managers, berth planners, vessel planners and yard planners were the key experts that were approached to collect opinions and current theories. All the loading, discharging and transshipment processes are dependent on the quay-to-yard interface. The loading process has the highest priority in the quay-to-yard interface. Yard-to-gate operations are considered only after considering loading and discharging in yard priorities. In other words, quay operation is the prioritized operation over other operations. The yard operation is the primary, and one of the most complicated, functions that can be adjusted based on quay operations. Therefore, the focus of the study has been narrowed down to the quay-to-yard interface.

Alterations of the initial plan were identified as the key issue and the uncertain dynamic environment was identified as the main cause based on the discussions that were conducted with experts. When it comes to the time horizon, $12 \mathrm{~h}$ working shifts were identified due to two reasons: this time horizon aligns with the operational norm of the terminal and understanding uncertainties such as crane breakdowns was cumbersome when dealing with weekly, monthly or annual operations horizons. The hourly operation was the best time horizon to understand the crane breakdowns and their impact. However, every decision has a tradeoff. This identified time horizon is not the ideal time frame for evaluating the alterations done by the berth planner (Böse, 2020). Simultaneously, the changes effected by the berth planner which are included in the plan by the vessel planner and the yard planner within this time horizon are identified. 
MABR

8,1

70

The second step of SD was to identify the dynamic hypotheses. All the operational processes were integrated into one system through process mapping. The planned operations and actual operations were evaluated for each hour. The difference between the planned and the actual operation was calculated using mean absolute percentage error (MAPE) values as Table 1 shows. Table 1 indicates those alterations were balanced between hourly operations.

It does not matter whether the change is a negative or a positive one, the planner must incorporate it. Therefore, if the hourly changes are just added, the contribution of the planners is not calculated. "The changes are balanced within hourly operations and total alteration value that is calculated at the end of the shift does not emphasize the contribution from planners" is the first dynamic hypothesis that was developed. When the collected data points were carefully observed, it indicates that "the total MAPE value has been balanced between alterations in separate loading and discharging processes". That was the second dynamic hypothesis. Since the loading process has priority over the discharging process, the findings show that loading alterations were balanced by the discharging alterations. Then the key variables of the selected problem were identified as the second sub-step. Understanding the planning task through observing the task, planning manuals and standard operating procedures was the key methodological strategy employed. Main variables include actual and planned terminal productivities, hourly alterations by the planners, breakdown hours and so on. The most important part of SD generating dynamic hypotheses is the mapping part. However, some studies have deviated from the original steps that have been introduced by Sterman (2000). Bahadir and Akdag (2019) and Balachandra et al. (2020) have done their studies by introducing the mapping part into the main steps. This has become a common practice in SD due to the importance of mapping. However, the original SD steps that have been introduced by Sterman (2000) were followed for this study. Step 3 and 4 of SD will be discussed in the analysis (Section 4) and Step 5 will be discussed under discussion (Section 5). Vensim (version 8.0.9), which is one of the most recognized software used in SD research, was employed for this study (Soares and Neto, 2016).

\section{Analysis}

The first two steps of SD were presented under section 3. The remaining steps of SD are discussed under this section. The integrated process was observed carefully and 50 variables applicable for developing CLD were identified. All the variables were developed from the initial identification of the key variables part (section 3.2). Using the same process that was used to identify the initial dynamic hypotheses, the links were defined in a CLD as per Figure 2.

\subsection{Causal Loop Diagram (CLD)}

Polarities are very critical in CLDs since it classifies the causal links and causal loops. In causal links, it is easy to understand the theory, and the polarity represents the impact from one variable on one another. As Figure 2 explains, hourly remaining QC moves contribute in the same direction as expected terminal productivity contributes since the polarity is positive $(+)$. At the same time, actual terminal productivity affects hourly remaining moves negatively (-). To explain further, when the expected productivity is increased, hourly

Table 1.

MAPE values (actual operation versus planned operation)

\begin{tabular}{lccc}
\hline MAPE values & Loading $(\%)$ & Discharging $(\%)$ & Total $(\%)$ \\
\hline 1. Total shift operation & 0.6 & 2.6 & 1.5 \\
2. Average hourly operation & 4.02 & 6.75 & 3.88
\end{tabular}


Decision

alterations at

container

terminals

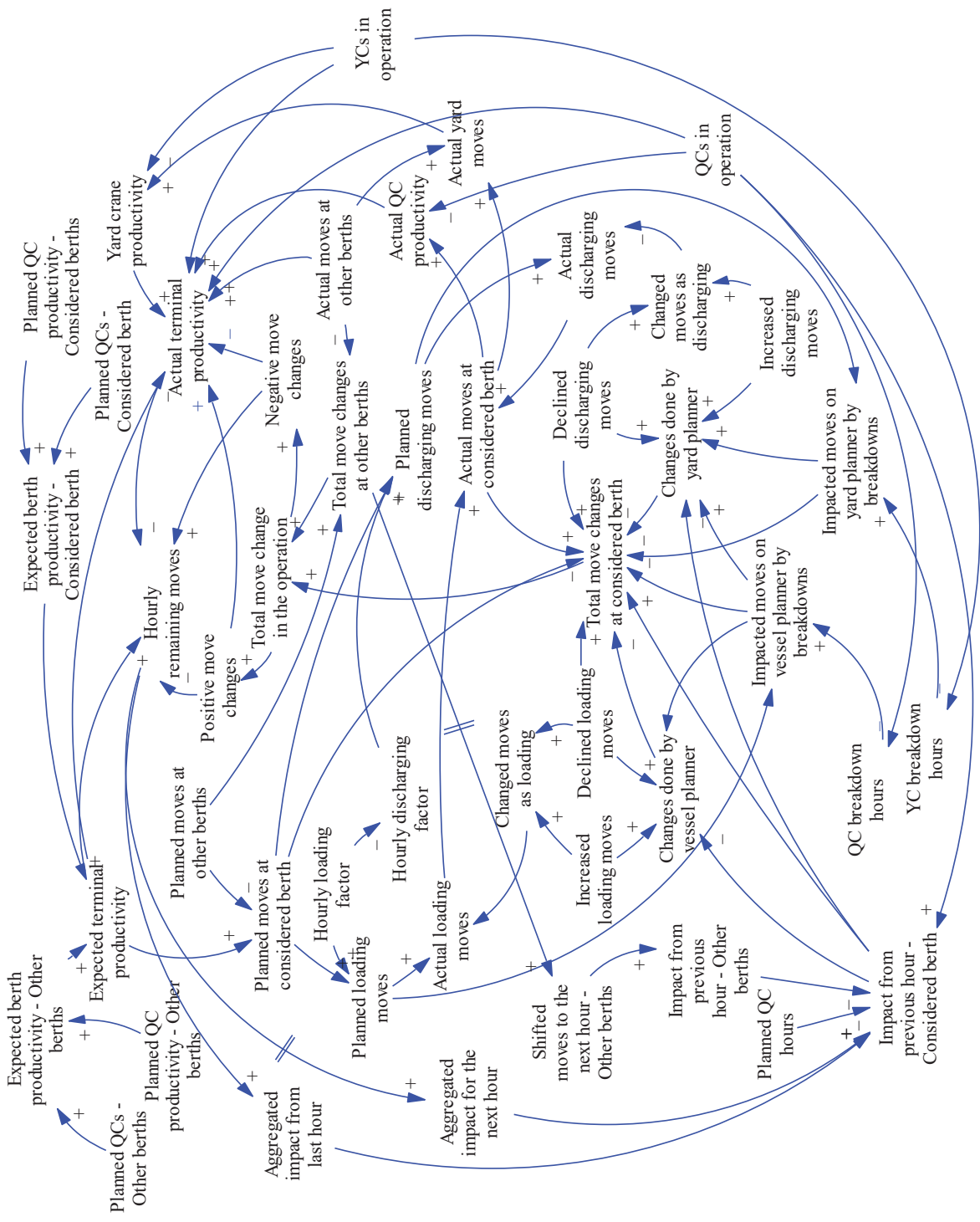

71

Figure 2.

CLD-planning alterations in container terminal operation 
MABR

8,1

\section{2}

remaining moves increase. When the expected productivity is decreased, the hourly remaining moves increase. In negative polarities, the inverse is performed. The polarity between the hourly loading factor and the hourly discharging factor is an example of negative polarity. It was identified that the main stock of the system is "hourly remaining stock" while the "expected terminal productivity" and "actual terminal productivity" are being performed as the main inflow and outflow of the system. The Stock and Flow diagram (SFD) was created based on this finding as illustrated by Figure 3 .

\subsection{Stock and Flow Diagram (SFD)}

As it was found that the yard moves are dependent on QC moves (points of working in quayside.), expected terminal productivity was defined based on $\mathrm{QC}$ productivity levels at each berth and number of QCs that were deployed. However, yard moves should be analyzed since yard moves should be performed in loading and discharging operations. Therefore, the actual terminal productivity is calculated based on both the yard and quay performances overseeing the actual number of moves. According to Sterman (2000), stock can be identified when a variable is influenced by two other variables in two directions ("+" and "-"). Additionally, it should be realistic as well. The same behavior was identified with "hourly

Figure 3.

SFD-planning alterations in container terminal operation

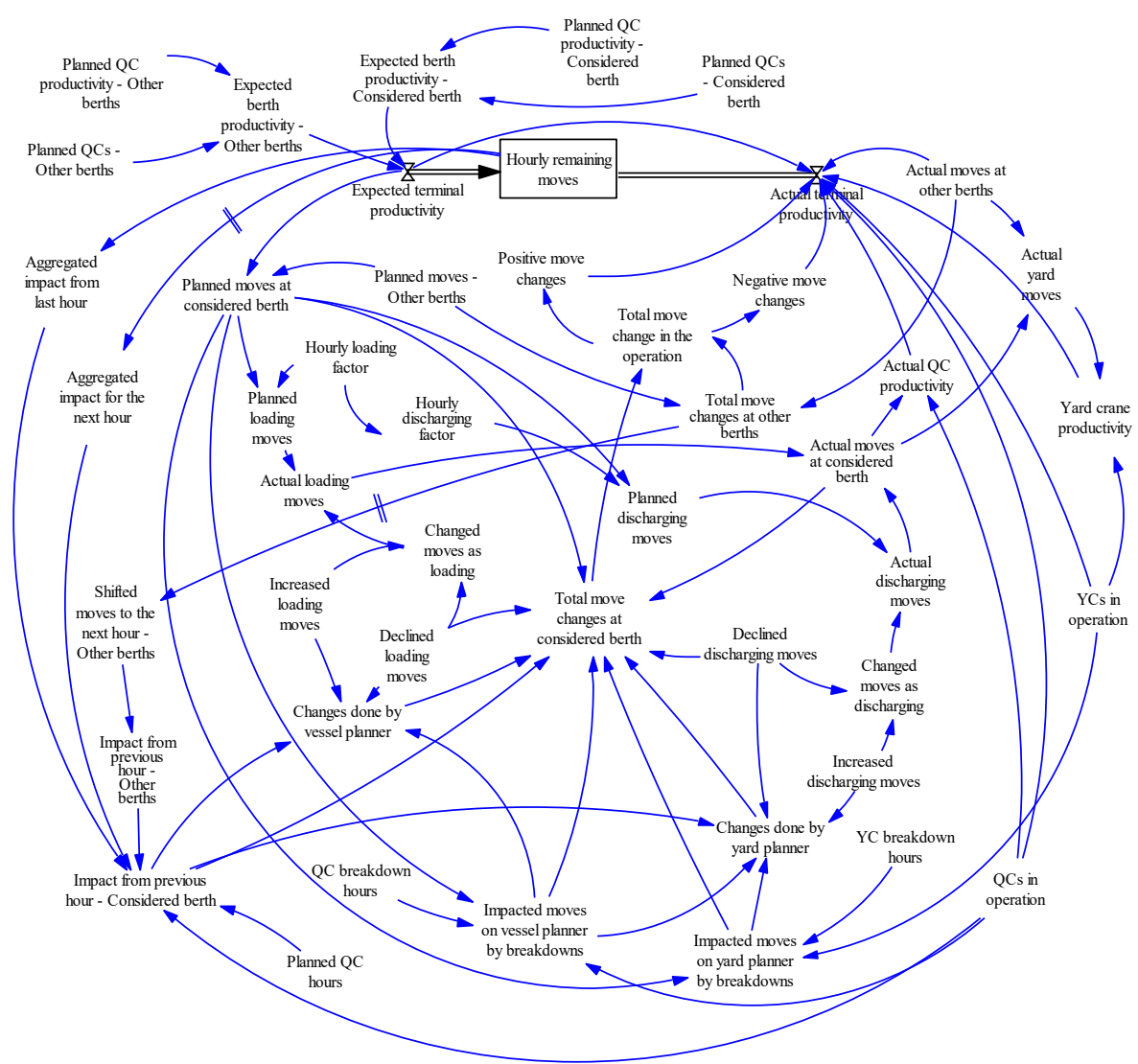


remaining moves", and it was defined as the main stock of the model. Equations 1 and 2 introduce the main formula of the stock and flow diagram.

Equation 1. Integral equation: planning alterations in container terminal planning

$$
\begin{aligned}
\text { hourly remaining moves }(t)= & \int_{\text {to }}^{t}[\text { expected terminal productivity }(s) \\
& - \text { actual terminal productivity }(s)] \\
& + \text { hourly remaining moves }\left(t_{0}\right)
\end{aligned}
$$

Equation 2. Differential equation: planning alterations in container terminal planning

$$
\frac{d \text { (hourly remaining moves })}{\mathrm{dt}}=\text { net change in stock }
$$

net change in hourly remaining moves $=$ expected terminal productivity $(t)$

$$
\text { - actual terminal productivity }(t)
$$

Both the equations have been derived based on Sterman (2000). All the equations were defined in the model and it has been formulated as the third step of SD modeling. Before testing the model, it should be verified by checking whether necessary components have not been overlooked. Names of the variables, unit check, time horizon and appropriate flow integration with stocks are checked before simulating the model. The "check unit" and "model check" features were used to validate the model.

The model testing was conducted as the fourth step of SD modeling. There are three types of testing in SD: direct structure tests, structure-oriented behavior tests and behavior reproduction tests (Bahadir and Akdag, 2019). Boundary adequacy test to check boundaries, structure assessment to see whether strict rules in the planning system have been applied and extreme condition test to see whether the model is accurate when extreme points are applied, are performed under direct structure tests. Behavior anomaly test was performed under structure-oriented behavior test to find whether the model displays any anomalous behavior. The reproducibility of the model was checked as the final part of testing to check whether the model returns no errors when the time is integrated.

\section{Results and discussion}

\subsection{Reinforcing and balancing loops}

A SD model can be explained using two types of loops based on their polarity: reinforcement loops and balancing loops (Sterman, 2000). Twelve loops were identified in the model: six reinforcement loops and six balancing loops. The factor that divides the loops into these categories (reinforcement and balancing) was found as the polarity of the hourly alterations which is indicated by positive move changes and negative move changes. Three main critical points were identified within loops. The first point is the aggregation of the time factor. The hourly remaining moves enter the system as the previous hourly operation itself. It is calculated at the end of each hour, and it adds to the next hour's operation where a delay function was identified in our SD model. The second point is whether there is a planner contribution or not. That means if the planner does not make alterations, still the initial plan can be performed. The direct link between "Impact from previous hour - Considered berth" and "Total move changes at considered berth" as displayed in Figure 4 is activated in this scenario. 
MABR

8,1

74

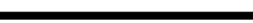

Figure 4.

Identified reinforcing and balancing loops
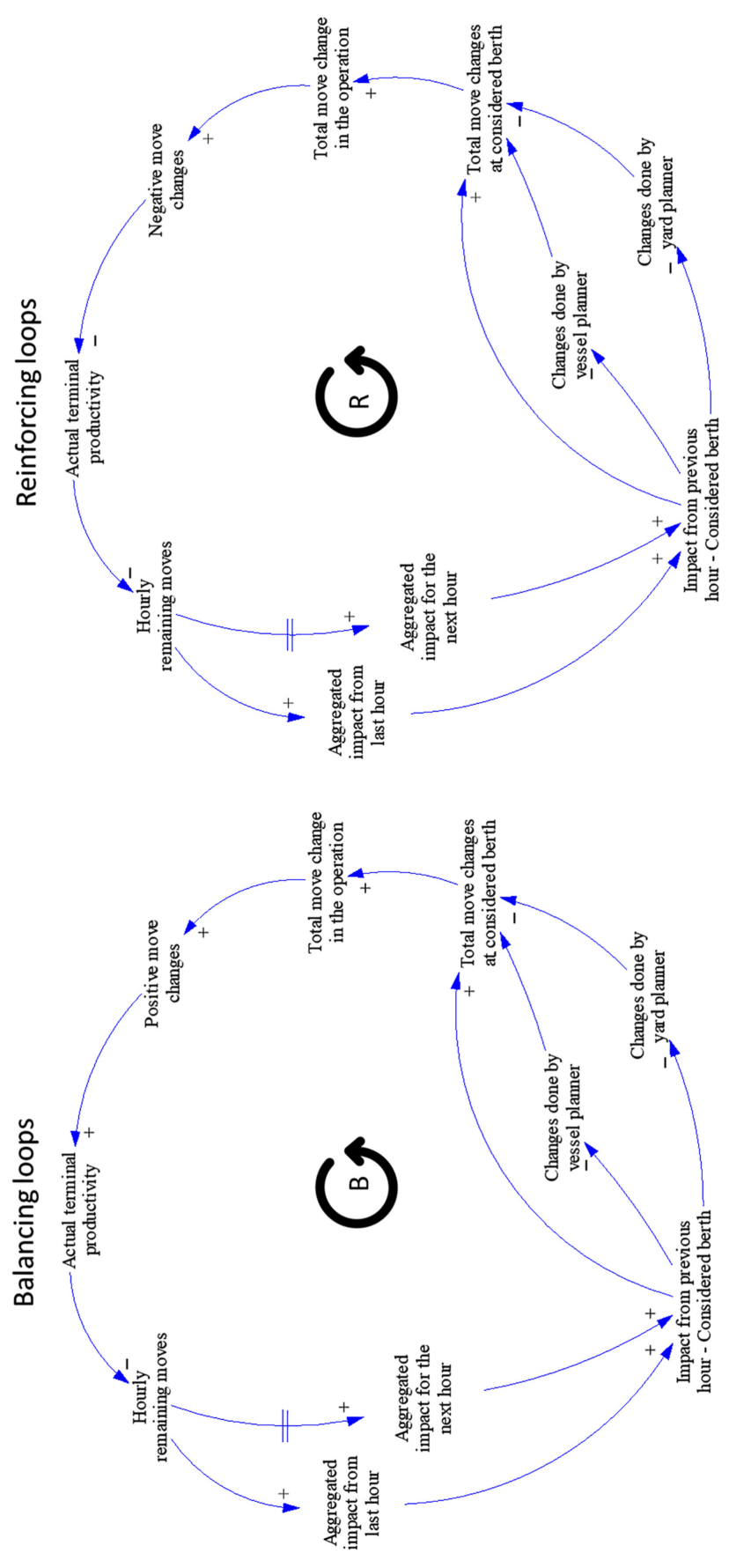
Subsequently, the initial plan goes through the vessel planner or yard planner. According to the dynamic hypotheses that were built when the model was created, their target is to increase terminal productivity by making their contribution as a positive change. Balancing loops are activated when positive changes occur. Reinforcement loops are activated when negative move changes occur. This aligns with the fundamentals of mathematics and when there is no difference between expected and actual productivities, still the balancing loop is activated through positive move changes.

The aggregated impact from the last hour adds to the system as the impact from the previous hour. At the same time vessel planner works on the plan aiming to reduce the impact for the next hour. Since the planner's objective is to reduce the overall impact on the initial plan, that reduces the number of alterations in that considered berth. However, if the total number of alterations (from all the berths) is positive, the actual terminal productivity increases, and it reduces the hourly remaining moves stock. This is an example of a balancing loop. If the total number of alterations is negative, the actual terminal productivity decreases and it increases the hourly remaining stock. This is an example of a reinforcing loop.

\subsection{Scenario analysis}

5.2.1 Crane breakdowns versus planner involvement. The breakdown levels and the planned move levels changed in the model and the results were observed through simulated values. Breakdown levels for both the QCs and YCs were increased and the planner alterations were observed. When the QC breakdown hours reach half of the allocated QC option the vessel planner has increased the number of alterations by $94 \%$, while the yard planner has increased alterations by $75 \%$. This indicates that the yard planner works on both $\mathrm{QC}$ and $\mathrm{YC}$ breakdowns while the vessel planner only focuses on $\mathrm{QC}$ breakdowns. Therefore, yard planner changes were observed when the $\mathrm{YC}$ breakdown level is increased. As a result, the yard planner has increased the breakdown level twice compared to the no breakdown scenario when the YC breakdown level reaches $8 \mathrm{~h}$.

5.2.2 The impact from other berths to a considered berth. The input points of expected berth productivities at other berths were changed in the initial model to identify the impact on a considered berth. When the planned workload has been reduced in other berths, the productivity of the considered berth increased since the pressure on the vessel planners in the considered berth was reduced. When the workload has been increased in other berths it has affected negatively on the considered berth. The highest variation was found with the yard planner. When the expected productivities are increased in other berths from $0 \%$ to $40 \%$, changes that have been made by the yard planner have increased from $0 \%$ to around $13 \%$. The impact of the yard planner on the considered berth has increased approximately by twofold when the alterations done by the vessel planner were observed. When the expected productivities at other berths have been reduced by $0-40 \%$, the impact on the yard planner has reduced between $0 \%$ and $13 \%$ while it was in a range between $0 \%$ and $7.3 \%$ for the vessel planner. These findings indicate why the dynamic approaches should be considered within the quay-to-yard interface. Conventional approaches can identify causes only within a task while dynamic approaches can showcase real causes within an interface or beyond as an impact.

\subsection{Findings}

This research was conducted with four objectives in mind. All the objectives were based on the integrated planning function that was modeled using planning alterations. The first research objective is to identify the significance of crane breakdowns in altering planning decisions. Both QC and YC breakdowns were investigated. Findings show that the operation does not stop once a breakdown occurs. First, the planner starts working on it using

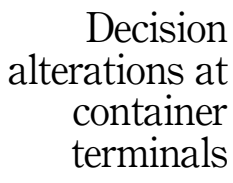


MABR

8,1

alteration. Contingency plans are created to solve the situation. The vessel planner involvement increases by $63 \%$ while yard planner increases alterations by $50 \%$ when the QC breakdown level reaches $1 \mathrm{~h}$. Yard planner alterations increase twice from the nonbreakdowns level when the YC breakdown reaches $8 \mathrm{~h}$.

Understanding the dynamic planning role within altering planning decisions was the second research objective that was derived under subsection 2.6. According to the causal loops, there are three critical points and planner contribution is one of them (subsection 5.1). Three paths determines whether the loop is reinforcing or balancing within that critical point as described in Figure 4. It is clear that the planner alters the plan when a breakdown occurs. An alternative plan must be created by the planner to sustain the operation. If the engineering team confirms that the breakdown continues for more than $1 \mathrm{~h}$, the planner must go for an alternative plan in which the relevant QCs or YCs are not included. This finding is very critical in redesigning the policies which is the final step of SD. The current policy is to keep the breakdown QC in the plan as it was identified in the interviews. When other berths changed their expected productivities, the planner tasks were observed alterations in a considered berth. It explains why the workload of the yard planner is higher than the vessel planner.

Understanding the impact of altering the initial plan on terminal efficiency was the last objective that was derived in the study. As it was discussed earlier, a critical point was identified through the CLD that decides the overall change of the initial plan. It depends on the overall change which is positive or negative. If the change is negative, it creates a reinforcement loop that motivates the remaining moves stock. The created remaining moves stock influences operations in the upcoming hour. If the change is positive, it reduces the remaining stock while activating the balancing loop. This is the exact scenario which the terminal operators are experiencing in real operations according to their feedback. As it was discussed under the results explaining the second objective, even though when a berth increased their productivity still other berths can perform below the expectation by reducing the overall actual terminal productivity.

At the same time even though the planner tries to create a positive impact through alterations it does not assure creating a balancing loop where the remaining move stock is reduced. It was found that 97 moves were unable to be facilitated when the expected productivity was not reached. Simply, it can be explained through reinforcement loops that were discussed within the overall impact according to the given scenario that the data points were collected. Annually, this amount accumulates to a considerable 70,810 moves. The salient point is when the vessel operator experiences this impact of reinforcement repeatedly, they might shift their calls to other terminals heavily impacting the relevant terminal operator. Therefore, the terminal operator must focus on the planning function as an integrated system to mitigate the impact on the imbalance between expected productivity that they promised to vessel operators and actual productivity that was delivered. In a nutshell, the SD model that is introduced in this study, explains all the causes that create planner alterations and their impact. The impact of crane breakdowns on the effort of the planners within the integrated planning function is explained avoiding the traditional way of explaining the integrated planning function using separated planning tasks.

\section{Conclusion}

This paper has investigated the issues that occur due to altering the initial operations plan in container terminals. This problem was discussed based on three directions. The first focus was the integrated planning function in container terminals. The second was the role of the planner. The last consideration was the uncertainty factor in terminal operations due to crane breakdowns. SD was used as the method to illustrate, model, test and evaluate the planning alterations. The model was built to illustrate this integrated function and how it works in 
dynamic reality. The feedback that is created by the gap between actual and expected terminal productivities was identified with relevant reinforcing and balancing loops. According to the findings, crane breakdowns alter the plan and planners engage in adjusting the initial plan. The workload among planners was not equally distributed and the yard planner works on crane breakdowns twice as much as the vessel planner. A successful plan is created when the planners are able to activate the identified balancing loop which demotivates hourly remaining moves stock through positive changes. When a berth is performing beyond the expected productivity levels, it does not assure a successful operation within the integrated system. As a result, it has a negative impact on other berths which means the terminal operator must focus on integrated planning, rather than separate working points for operational excellence.

Understanding the berth planning function with the role of berth planner by expanding the time horizon of the model, expanding the endogenous focus to the yard-to-gate interfaces and testing the feasibility of new methods to evaluate planners rather than depending on QC and YC moves which are implemented by crane operators will be potential future research directions. Concurrently, future research on port operations can consider applying SD as a methodology to better understand and explain the practical problems in terminals through an academic lens.

\section{References}

Bahadir, M.C. and Akdag, H.C. (2019), "The system dynamics modelling for container capacity \& transportation planning policies", Asian Journal of Shipping and Logistics, Elsevier B.V., Vol. 35 No. 4, pp. 200-212, doi: 10.1016/j.ajs1.2019.12.007.

Balachandra, K., Perera, H.N. and Thibbotuwawa, A. (2020), "Human factor in forecasting and behavioral inventory decisions: a system dynamics perspective", International Conference on Dynamics in Logistics (LDIC), Springer International Publishing, pp. 516-526, doi: 10.1007/978-3030-44783-0_48.

Beens, M.A. and Ursavas, E. (2016), "Scheduling cranes at an indented berth", European Journal of Operational Research, Elsevier B.V., Vol. 253 No. 2, pp. 298-313, doi: 10.1016/j.ejor.2016.02.038.

Böse, J.W. (2020), "Handbook of terminal planning", Operations Research/Computer Science Interfaces Series, Vol. 49, doi: 10.1007/978-1-4419-8408-1.

Chen, J.H., Lee, D.H. and Cao, J.X. (2012), “A combinatorial benders' cuts algorithm for the quayside operation problem at container terminals", Transportation Research Part E: Logistics and Transportation Review, Elsevier, Vol. 48 No. 1, pp. 266-275, doi: 10.1016/j.tre.2011.06.004.

Dadashi, A., Dulebenets, M.A., Golias, M.M. and Sheikholeslami, A. (2017), "A novel continuous berth scheduling model at multiple marine container terminals with tidal considerations", Maritime Business Review, Vol. 2 No. 2, pp. 142-157, doi: 10.1108/MABR-02-2017-0010.

Dulebenets, M.A. (2017), "A novel memetic algorithm with a deterministic parameter control for efficient berth scheduling at marine container terminals", Maritime Business Review, Vol. 2 No. 4, pp. 302-330, doi: 10.1108/MABR-04-2017-0012.

Görges, M. and Freitag, M. (2020), "On the influence of structural complexity on autonomously controlled automobile terminal processes", International Conference on Dynamics in Logistics (LDIC), Vol. 2, pp. 42-51, doi: 10.1007/978-3-030-44783-0_4.

Gunawardhana, J.A., Perera, H.N. and Thibbotuwawa, A. (2021), "Rule-based dynamic container stacking to optimize yard operations at port terminals", Maritime Transport Research, Elsevier, Vol. 2, March, 100034, doi: 10.1016/j.martra.2021.100034.

He, J., Tan, C. and Zhang, Y. (2019), "Yard crane scheduling problem in a container terminal considering risk caused by uncertainty", Advanced Engineering Informatics, Elsevier, November 2018, Vol. 39, pp. 14-24, doi: 10.1016/j.aei.2018.11.004.

\section{Decision alterations at container terminals}


MABR

8,1

Jayalath, M.M. and Perera, H.N. (2021), "Mapping post-harvest waste in perishable supply chains through system dynamics: a Sri Lankan case study”, Vol. 16 No. 3, pp. 144-161, doi: 10.4038/jas. v16i03.9476.

Jin, J.G., Lee, D.H. and Cao, J.X. (2014), "Storage yard management in maritime container terminals", Transportation Science, Vol. 50 No. 4, pp. 1300-1313, doi: 10.1287/trsc.2014.0527.

Kavoosi, M., Dulebenets, M.A., Abioye, O., Pasha, J., Theophilus, O., Wang, H., Kampmann, R. and Mikijeljević, M. (2019), "Berth scheduling at marine container terminals: a universal islandbased metaheuristic approach", Maritime Business Review, Vol. 5 No. 1, pp. 30-66, doi: 10. 1108/MABR-08-2019-0032.

Kizilay, D. and Eliiyi, D.T. (2020), "A comprehensive review of quay crane scheduling, yard operations and integrations thereof in container terminals", Flexible Services and Manufacturing Journal, Springer US, Vol. 33, pp. 1-42. doi: 10.1007/s10696-020-09385-5.

Kurapati, S., Lukosch, H., Eckerd, S., Verbraeck, A. and Corsi, T. (2017), "Relating planner task performance for container terminal operations to multi-tasking skills and personality type", Transportation Research Part F: Traffic Psychology and Behaviour, Vol. 51, pp. 47-64, doi: 10. 1016/j.trf.2017.09.002.

Legato, P. and Mazza, R.M. (2019), "Queueing analysis for operations modeling in port logistics", Maritime Business Review, Vol. 5 No. 1, pp. 67-83, doi: 10.1108/MABR-09-2019-0035.

Legato, P. and Monaco, M.F. (2004), "Human resources management at a marine container terminal", European Journal of Operational Research, Vol. 156 No. 3, pp. 769-781, doi: 10.1016/S03772217(03)00134-6.

Lindroth, E., Huong, H. and Bergqvist, R. (2020), "Port-related conflict at port of Gothenburg consequences from a fashion retailer's perspective", Journal of Shipping and Trade. doi: 10. 1186/s41072-020-00059-x.

Mar-Ortiz, J., Castillo-García, N. and Gracia, M.D. (2019), "A decision support system for a capacity management problem at a container terminal”, International Journal of Production Economics, Elsevier B.V. doi: 10.1016/j.ijpe.2019.09.023.

Navis LLC (2014), Navis N4 XPS Vessel Planning and Control, Navis.

Nishimura, E. (2019), "Yard and berth planning efficiency with estimated handling time", Maritime Business Review, Vol. 5 No. 1, pp. 5-29, doi: 10.1108/MABR-08-2019-0034.

Pham, T.Q.M., Park, G.K. and Choi, K.H. (2020), "The efficiency analysis of world top container ports using two-stage uncertainty DEA model and FCM", Maritime Business Review, Vol. 6 No. 1, pp. 2-21, doi: 10.1108/MABR-11-2019-0052.

Soares, C.J.M. and Neto, H.X.R. (2016), "A model for predictable capacity of a container terminal state: a system dynamics approach”, Journal of Traffic and Transportation Engineering, Vol. 4 No. 3, pp. 141-154, doi: 10.17265/2328-2142/2016.03.003.

Stahlbock, R. and Voß, S. (2007), "Operations research at container terminals: a literature update”, $O R$ Spectrum, Vol. 30 No. 1, pp. 1-52, doi: 10.1007/s00291-007-0100-9.

Sterman, J. (2000), Systems Thinking and Modeling for a Complex World, available at: http://www. lavoisier.fr/notice/frJWOAR6SA23WLOO.html.

UNCTAD (2019), The Review of Maritime Transport 2019, United Nations, available at: https:// unctad.org/en/PublicationsLibrary/rmt2019_en.pdf.

UNCTAD (2020), Review of Maritime Transport 2020, Vol. 53, available at: https://unctad.org/system/ files/official-document/rmt2020_en.pdf.

Zhen, L., Xu, Z., Wang, K. and Ding, Y. (2016), "Multi-period yard template planning in container terminals", Transportation Research Part B: Methodological, Elsevier, Vol. 93, pp. 700-719, doi: 10.1016/j.trb.2015.12.006. 
Zhou, Y., Wang, W., Song, X. and Guo, Z. (2016), "Simulation-based optimization for yard design at mega container terminal under uncertainty", Mathematical Problems in Engineering, Vol. 2016, doi: $10.1155 / 2016 / 7467498$.

\section{About the authors}

Buddhi A. Weerasinghe is an MSc student and Research Assistant at the Center for Supply Chain, Operations and Logistics Optimization, University of Moratuwa, Sri Lanka. Buddhi is also affiliated with the Professor H.Y. Ranjit Perera Institute for Applied Research, Sri Lanka. His MSc focuses on container terminal optimization. Buddhi holds a BSc (Hons) degree in Transport and Logistics Management from the Faculty of Engineering, University of Moratuwa, Sri Lanka. Ports, maritime logistics, container terminals, port management and port optimization are his main research interests.

H. Niles Perera, PhD, is the Founding Director of the Center for Supply Chain, Operations and Logistics Optimization at the University of Moratuwa, Sri Lanka. Niles is an award-winning graduate of the Department of Transport and Logistics Management who graduated as the Most Outstanding Graduand of the University of Moratuwa, Sri Lanka. He holds a Graduate Certificate in Business Administration from the Australian Institute of Business and a $\mathrm{PhD}$ on Supply Chain Optimization from the University of Sydney, Australia. Niles' research has appeared in journals such as European Journal of Operational Research, Energy Policy, International Journal of Operations and Production Management and Maritime Transport Research. His primary research interests lie in behavioral operations and supply chains, energy supply chains and maritime logistics. H. Niles Perera is the corresponding author and can be contacted at: hniles@uom.lk; hnp@hyrp.lk

Phillip Kießner is a $\mathrm{PhD}$ candidate at the Chair of Enterprise Logistics at Technical University of Dortmund, Germany. The focus of his research is concentrated in complexity and logistics management. He has completed his MSc at the Institute of Transport and Logistics at TU Dortmund, Germany. His primary research interests lie in operations research, supply chains and maritime logistics.
For instructions on how to order reprints of this article, please visit our website: www.emeraldgrouppublishing.com/licensing/reprints.htm Or contact us for further details: permissions@emeraldinsight.com

\section{Decision alterations at container terminals}

